\title{
The gnosis of knowledge navigation on the Net
}

\author{
ERIK NILSEN \\ Lewis and Clark College, Portland, Oregon
}

\begin{abstract}
A set of activities developed to expose liberal arts undergraduates to global networking software and resources is detailed. Using a suite of Internet software tools and Macintosh computers, teams of students collaborated with faculty searching the World-Wide Web for on-line resources. Detailed student logs provide insights into the challenges of searching for Internet resources. Project shortcomings include difficulty in accessing information sites and poor organization of the final written reports. Ideas for improving the project in the future include allowing more time for the search process and replacing the written report with a WWW home page. The class project enhances the utilization of technology in the curriculum while equipping the students with computer skills to enhance their education.
\end{abstract}

This paper describes a term project that students were required to complete as part of class at a liberal arts college. The course, Psychology 320: Human-Computer Interaction, has two goals. The first is to expose students to the wide range of psychological issues that are involved in the use of computers. Course readings are primarily taken from the proceedings of the Association of Computing Machinery's (ACM) yearly CHI conferences on Human Factors in Computing Systems (ACM, 1983-1995). Major course topics surveyed include (1) user classification and individual differences, (2) performance modeling, (3) input/output devices, (4) computer-supported cooperative work (CSCW), (5) virtual reality, and (6) global networking. The second goal of the course is to explore one topic in depth, combining experiential learning with the reading of the latest research results and technological advances. For the past 3 years the focus of the course has been on CSCW. A fuller description of this topic can be found in a paper entitled "Computer Supported Cooperative Work Meets the Liberal Arts" (Nilsen, 1993).

The focus of the fall 1994 course was the use of the software tools Gopher, Fetch, and Mosaic/MacWeb to explore the rapidly growing computer resources available on the Internet and the World-Wide Web (WWW). An in-depth overview of Internet resources can be found in Krol (1994). Steen (1995) has provided some additional case studies of the use of the Internet in teaching. The goal of the activities described here was to structure students' introduction to global networking so that they become successful searchers for the "esoteric knowledge" (gnosis) of cyberspace.

\section{METHOD}

\section{Student Background}

Twenty-eight juniors and seniors enrolled in the class. Fifteen of them were psychology majors, with the remainder majoring in

Correspondence should be addressed to E. Nilsen, Lewis and Clark College, 0615 SW Palatine Hill Road, Portland, OR 97219 (e-mail: nilsen@lclark.edu; WWW address: http://www.lclark.edu/ nilsen). art, biology, business, international affairs, mathematics, English, and physics. The course fulfilled a major elective requirement for the psychology majors and a general education requirement for the non-psychology majors. All of the students had prior experience using a Macintosh computer for word processing. Two thirds of them had used electronic mail. Only 3 students had computer science backgrounds, so one challenge of the course was to present the material from a nontechnical perspective.

\section{Classroom Setup}

The classroom environment consisted of tables and chairs arranged in a square in the middle of the room. Ten Macintosh IIcx and five IIsi computers were placed along the walls. The teaching computer was a Quadra 840AV connected to a 32-in. TV to display the computer screen to the class. A Macintosh Quadra 700 served as a dedicated file server. The computers were connected via $10 \mathrm{base} T$ ethernet to the rest of the campus, which is connected to the Internet through a $\mathrm{T} 1$ connection.

\section{Description of Software}

Three programs form the core of the set of tools used for exploring the Internet. The first one is Gopher, accessed through the user accounts on the campus mainframe. The second program is Fetch, which provides a menu-driven interface for downloading files from remote computers using file transfer protocol (FTP). The evolution of the WWW browser software used in the course reflects the rapid rate of change in networked computing. From the time the course was initially conceptualized in the spring of 1994 until the end of the class, NCSAMosaic evolved from release 1.03 to 2.08 (Web, 1; see Appendix). During the semester, two additional WWW browsers became available. MacWeb (Web, 2), and Netscape (Web, 3) both provided quicker and more reliable access to WWW sites than the extant version of NCSAMosaic. Students were introduced to these browsers as they became available. The class chose MacWeb as the browser to use for the knowledge navigation project.

\section{Preparatory Exercises}

Geographic Gopher search. On the 1st day of class, students were introduced to the global scale of the Internet through the use of Gopher. Groups of 3 students each were given a geographic area of the world to explore using the University of Minnesota directory of Gopher servers (Web, 4). They were given post-it notes to record the names of the countries that had Gopher servers and to write the number of sites in each country. After $30 \mathrm{~min}$, they placed the post-it notes on a large world map on one wall of the 
classroom. The appearance of the map and the numbers gave them a good visual gestalt of the geographic distribution of the Internet. North America and Europe were covered with paper, with another cluster in the Far East. The countries with the largest number of Gopher sites were the United States, with 1,366; Canada, with 122; United Kingdom, with 105; France, with 41; Japan, with 38; and Germany, with 37 . In contrast, only two countries were represented in the continent of Africa: South Africa, with 14; and Tunisia, with a single Gopher site. This project did a good job of impressing on the students' minds the scale and geographical distribution of the Internet.

WWW browser training. One class session early in the term was devoted to learning to use NCSAMosaic. To cement their learning and prepare them for the knowledge navigation project, two take-home assignments were given over the next 2 weeks of class. The first assignment was to simply browse through various home pages, including the What's New page (Web, 5), and report back on interesting treasures that they found.

The second assignment required a more focused search on a specific topic. The entire class searched for information regarding adaptive speech technology for the handicapped. Individual students also selected his/her own topic ahead of time. To aid students in their search, they were given a "search hotlist" of several home pages that support searches of various kinds, including keyword search and subject hierarchies. The search pages included ArchiePlexForm (Web, 6), Veronica (Web, 7), EInet Galaxy (Web, 8), and Big Wide World Connections (Web, 9), which gives a comprehensive list of other search home pages.

The student reports on this assignment highlighted the difficulties of searching for specific information on the WWW. Some of the searches were highly successful, but nearly half of the class did not find exactly what they were looking for. There were several problems in using the search home pages. These included difficulty in accessing them ("too busy, try again later"), a high ratio of irrelevant home pages in the search results, and some of the searches aborting and causing the computer to freeze. The class discussion revolved around how to achieve the right level of specificity in composing the search and which search mechanisms were the most reliable.

The clear favorite of all of the search home pages was one provided by MCC called EINet Galaxy (Web, 8). This home page provides two ways to search for information; an extensive categorized menu of topics and a keyword search mechanism. The menu system is ideal for getting the feel for what is available on the Web for users with limited knowledge of the subject domain. The hierarchical structure serves as a guide to focus the search in a logical manner rather than following sometimes arbitrary links among various home pages. The keyword search is especially useful for navigators who have a working knowledge of the important terms for the subject domain.

\section{Knowledge Navigation Project}

Selection of project. Following the preparatory exercises, the students began the central research project of the course. Teams of 2-5 students worked with a faculty member to formulate a search for freely available resources on the Internet and the WWW. Faculty were recruited to try to provide a representative sample of the academic disciplines at the college. Students were allowed to select the area they were interested in from a list of options provided by the instructor. The following is a list of departments and topic areas that were selected: biology, endangered plant species in the Pacific Northwest; East Asian studies, 20th-century Japanese history and popular culture; education, cooperative learning; foreign language lab, granting agencies and opportunities; Spanish, Hispanic women's testimonial literature; general education, inventing America (a required first-year course); psychology, brain and behavior (test batteries and diagnostic tools); psychology, cheetah (socialization and development).
Procedure. The search process was done in two phases. For the first phase, the faculty were asked to choose a subject area that they would use within the next year. It could be related to their teaching or research interests. Having selected a topic, they formulated a description of 3-5 sentences of the area and sent this to the student team by e-mail. The students used this short description to conduct a preliminary search on the Internet over the course of several days.

The faculty and students scheduled a face-to-face meeting to refine the topic area and focus the search. The students brought the "finds" of their preliminary search to the meeting. The faculty member brought artifacts to help the students understand the topic area, such as class syllabi, research articles, and names of people and professional organizations.

Following this meeting, the student team spent a week to 10 days "surfing cyberspace" for resources. The teams were allowed to organize their collaboration in their own manner. The only requirement was that they keep an accurate log of the time they spent searching, annotated with comments of when, where, and how they found relevant materials. These logs served as the record of the process of the search and were used to ensure individual accountability in the group project.

The final product of the search was a collaboratively written report and a collection of resources to be given to the faculty member. The report included a description of the resources and information on how to access them, a hotlist containing all WWW home pages, and any downloaded software on disk.

The faculty member then spent 1 week evaluating the resources. The faculty member then provided the group with one to two pages of written feedback on the quality of the "treasures" the students had found and the potential and planned uses for them.

\section{RESULTS}

The logs that the students kept provide some useful insights into the process of searching for Internet resources. They spent an average of $9 \mathrm{~h}$ actually searching the Internet. Over $7 \mathrm{~h}$ of this time was spent browsing the WWW. A few individuals started out by using Fetch to browse file transfer protocol (FTP) sites. This approach was quickly abandoned, as the sparse information provided by this protocol does not work well unless you know the names and locations of the files for which you are searching. Several people successfully used Fetch to download software located through other information sources. Not 1 of the 28 students used the Gopher system provided on the campus mainframe computer. This was somewhat surprising since their initial exposure to Internet resources on the 1st day of class utilized this program. The ability to access many of the Gopher sites using MacWeb undoubtedly led to abandonment of the mainframe version of Gopher. Ten of the students used a Macintosh Gopher program called Turbogopher, which was available in the campus computer labs.

The logs revealed that approximately $60 \%$ of the time students spent using the WWW browser, MacWeb, was with the EINet Galaxy (Web, 8) home page discussed earlier in the paper. Six of the students utilized only the categorized menus to guide their search for relevant material while 18 used both the menus and the keyword search mechanism. The students spent the rest of the time following links that they found serendipitously while browsing Web pages. 
The students' success in locating relevant information on the faculty members' topics was decidedly mixed. Among the most successful groups were the ones studying (1) education (cooperative learning) and (2) general education (inventing America). The education group found an enormous amount of material ranging from U.S. Department of Education publications to on-line discussion groups to listings of all $\mathrm{K}-12$ schools that have WWW sites. The inventing America group likewise found a plethora of material on American history. In addition to locating on-line versions of famous historical documents, they found several sources of information that use the hypermedia capabilities of the WWW to its fullest. One notable example of this is a page called The Nation's Forum (Web, 10). This database contains both the audio and full text of 59 speeches by American political and business leaders at the turn of the 20th century. The database is searchable by topic and speaker.

The cheetah research group had the most difficulty in finding material. A search of all of the information sources came up with only one book reference and several pictures of cheetahs. After meeting with the faculty member, they switched their topic to social development of the great apes. They were successful in finding good sources of information on primates, including information on several primate research centers. However, when they narrowed the search to social development of the great apes, they again came up empty. Finally, they shifted the topic to the influence of teratogens on prenatal development in humans and came up with several references to off-line resources. It is clear from this group's experience that certain fields of inquiry are not yet well represented on the Internet.

Many of the groups had the similar experience of initially locating a large number of sites when exploring a general area, only to be disappointed at the dearth of material when they focused their search on a more specific topic. A useful analogy is an information sieve: At the aggregate level, the sieve holds a great deal. However, when the clumps of information are broken apart, the smaller particles fall through the holes of the sieve, leaving an empty container. Whether this problem is due to poor search mechanisms or to the early stage of development of the WWW is unclear.

Another problem that appeared in every $\log$ was the inability to access sites. Sometimes this was caused by the site limiting the number of concurrent users. If the capacity was overloaded, students got a "too busy, try again later" message. Occasionally the host computer was off-line or the site had been moved to a different computer with no forwarding address. At other times the program would freeze for no apparent reason when students were trying to connect to a home page. One frequent problem was with certain Gopher and FTP sites that were accessed through the WWW browser. Although the files and directories could be seen with the browser, they could not be accessed. In the fall of 1994, there were still some compatibility problems with the various Internet protocols. The student logs reveal very little patience with busy and inaccessible home pages. These sites were rarely tried again. In a similar vein, search pages that produced no hits on the first attempt were seldom revisited.

The final product of the project was a written report for the faculty member, summarizing the resources and providing addresses to potentially relevant sites. The hope was that these reports would be well-organized, cogent summaries of the material the teams had found. The reality was that on the whole, the reports resembled the chaotic nature of the Internet. Page after page of screen printouts and Internet addresses were the norm. The prose that accompanied the material was much more likely to describe the process used to obtain the material than to describe the content. To be fair to the students, this problem probably should have been foreseen. After all, the students have little or no domain knowledge going into the project. It stands to reason that they would present the information to the faculty in a "raw" form and emphasize the part of the project about which they felt most competent, the process of navigating the information space.

The best written report organized the information by source, including sections on e-mail lists and news groups, WWW home pages, and downloaded shareware. Each section was prefaced with step-by-step instructions on how to access the information. The paper was written collaboratively and had a consistent style. The two groups with the least impressive reports divided up the search task and worked independently. The final paper consisted of individual reports containing redundant information and lacking coherence.

Six of the eight faculty provided the groups with written feedback in a timely fashion. However, only one faculty member had actually used the Internet to explore the information that he had been given during the 1-week evaluation period.

\section{DISCUSSION}

Student response to the knowledge navigation project was enthusiastic. Students like the idea of using their developing skills on projects that have the potential to make a lasting contribution to the college. This sentiment was reflected in a reaction paper that each student wrote following the project. All 28 of the students felt that it was a valuable learning experience and that it should be incorporated into future classes. The rapid pace of change in available resources and searching tools contributes to a highly collaborative class setting where students take an active role in the learning process.

Faculty members were likewise enthusiastic about having students search for on-line material to aid their teaching and research. Without exception, they praised the students for their conscientious effort. It is too early to tell if and how the faculty will incorporate the material into their ongoing projects. One limiting factor is the level of experience that the professors have with the technology. One person had never logged into an e-mail 
account prior to this project. At the other extreme, one professor had used a WWW browser for 1 year and was in the process of developing a home page.

This project will be refined and repeated the next time this course is taught in the winter 1996 semester. One change will be in the amount of time spent on the project. Three weeks was clearly not enough time to do a thorough search and compile the data into a coherent, organized report. A minimum of 4 weeks is needed, with more frequent consultation with the faculty collaborator. Netscape will be the WWW browser used next time as it has become the de facto standard for a vast majority of WWW users.

Instead of a written final report, the groups will create a WWW home page containing a description of the resources found and hypertext links to relevant home pages. This will serve three purposes. It imposes a common structure to the report and helps to ensure that the students coordinate their efforts on a single document. It also provides a convenient format for the faculty members to evaluate the information with relatively little effort. The report will also be ready for the faculty members to modify and customize for their own use. This will provide tangible (albeit electronic) evidence of the contribution that the students have made to the academic life of the college.

\section{REFERENCES}

Association of Computing MaChinery (Ed.) (1983-1995). Conference on Human Factors in Computing (CHI Conference Proceedings). New York: ACM Press.
KROL, E. (1994). The whole Internet: User's guide and catalog (2nd ed.). Sebastopol, CA: O'Reilly \& Associates.

NILSEN, E. (1993). Computer supported cooperative work meets the liberal arts. Psychology Teaching Review, 2(2), 96-105.

STEEN, D. (1995). Teaching with the Internet: Putting teachers before technology, Resolution Business Press.

\section{APPENDIX}

The following are the uniform resource locator (URL) addresses for the WWW pages described in the text. They are presented in the order in which they appear in the article.

(Web, 1) NCSAMosaic: http://www.ncsa.uiuc.edu/SDG/Software/ Mosaic/NCSAMosaicHome.html

(Web, 2) MacWeb: http://galaxy.einet.net/EINet/MacWeb/ MacWebHome.html

(Web, 3) Netscape: http://home.netscape.com/

(Web, 4) University of Minnesota directory of Gopher servers: gopher://gopher.micro.umn.edu/11/Other\%20Gopher\%20and \%20Information\%20Servers

(Web, 5) What's New Homepage: http://www.ncsa.uiuc.edu/ SDG/Software/Mosaic/Docs/whats-new.html

(Web, 6) ArchiePlexForm: http://www.lerc.nasa.gov/archieplex/

(Web, 7) Veronica: gopher://veronica.scs.unr.edu/11/veronica

(Web, 8) EInet Galaxy: http://galaxy.einet.net/

(Web, 9) Big Wide World Connections: http://hakatai.mcli. dist.maricopa.edu/smc/ml/big-w-w-connections.html

(Web, 10) The Nation's Forum: http://lcweb2.loc.gov/nfhome. html

(Manuscript received November 13, 1995; revision accepted for publication January 18, 1996.) 